\title{
GLUT1 mutations are a cause of paroxysmal exertion-induced dyskinesias and induce hemolytic anemia by a cation leak
}

\author{
Yvonne G. Weber,1,2 Alexander Storch,1,3 Thomas V. Wuttke,1,4 Knut Brockmann, ${ }^{5}$ Judith Kempfle,1,4 \\ Snezana Maljevic,,$^{1,4}$ Lucia Margari, ${ }^{6}$ Christoph Kamm,, Susanne A. Schneider, ${ }^{8}$ Stephan M. Huber, ${ }^{9}$ \\ Arnulf Pekrun, ${ }^{10}$ Robert Roebling, ${ }^{1}$ Guiscard Seebohm, ${ }^{9}$ Saisudha Koka, ${ }^{9}$ Camelia Lang, ${ }^{9}$ \\ Eduard Kraft, ${ }^{1}$ Dragica Blazevic, ${ }^{1,4}$ Alberto Salvo-Vargas, ${ }^{1,4}$ Michael Fauler, ${ }^{4}$ Felix M. Mottaghy, ${ }^{11}$ \\ Alexander Münchau, ${ }^{8}$ Mark J. Edwards, ${ }^{8}$ Anna Presicci, ${ }^{6}$ Francesco Margari, ${ }^{12}$ Thomas Gasser, ${ }^{7}$ \\ Florian Lang, ${ }^{9}$ Kailash P. Bhatia, ${ }^{8}$ Frank Lehmann-Horn, ${ }^{4}$ and Holger Lerche ${ }^{1,4}$ \\ ${ }^{1}$ Neurologische Klinik and ${ }^{2}$ Institut für Anatomie und Zellbiologie, Universität Ulm, Ulm, Germany. ${ }^{3}$ Neurologische Klinik der Technischen Universität Dresden, \\ Dresden, Germany. ${ }^{4}$ nstitut für Angewandte Physiologie, Universität Ulm, Ulm, Germany. ${ }^{5}$ ädiatrie II mit Schwerpunkt Neuropädiatrie, \\ Georg-August-Universität Göttingen, Gottingen, Germany. ${ }^{6}$ Child Neuropsychiatric Unit, Department of Neurological and Psychiatric Sciences, \\ University of Bari, Bari, Italy. ${ }^{7}$ Neurologische Universitätsklinik, Neurologie mit Schwerpunkt Neurodegeneration Erkrankungen am Hertie-Institut \\ für Klinische Hirnforschung, Tubingen, Germany. ${ }^{8}$ Sobell Department of Motor Neuroscience and Movement Disorders, Institute of Neurology, \\ University College London, London, United Kingdom. ${ }^{9}$ Institut für Physiologie I, Universität Tübingen, Tubingen, Germany. \\ ${ }^{10}$ Pädiatrie I mit Schwerpunkt Hämatologie/Onkologie, Georg-August-Universität Göttingen, Gottingen, Germany. ${ }^{11}$ Klinik für Nuklearmedizin, \\ Universität UIm, Ulm, Germany. ${ }^{12}$ Psychiatric Unit, Department of Neurological and Psychiatric Sciences, University of Bari, Bari, Italy.
}

\begin{abstract}
Paroxysmal dyskinesias are episodic movement disorders that can be inherited or are sporadic in nature. The pathophysiology underlying these disorders remains largely unknown but may involve disrupted ion homeostasis due to defects in cell-surface channels or nutrient transporters. In this study, we describe a family with paroxysmal exertion-induced dyskinesia (PED) over 3 generations. Their PED was accompanied by epilepsy, mild developmental delay, reduced CSF glucose levels, hemolytic anemia with echinocytosis, and altered erythrocyte ion concentrations. Using a candidate gene approach, we identified a causative deletion of 4 highly conserved amino acids (Q282_S285del) in the pore region of the glucose transporter 1 (GLUT1). Functional studies in Xenopus oocytes and human erythrocytes revealed that this mutation decreased glucose transport and caused a cation leak that alters intracellular concentrations of sodium, potassium, and calcium. We screened 4 additional families, in which PED is combined with epilepsy, developmental delay, or migraine, but not with hemolysis or echinocytosis, and identified 2 additional GLUT1 mutations (A275T, G314S) that decreased glucose transport but did not affect cation permeability. Combining these data with brain imaging studies, we propose that the dyskinesias result from an exertion-induced energy deficit that may cause episodic dysfunction of the basal ganglia, and that the hemolysis with echinocytosis may result from alterations in intracellular electrolytes caused by a cation leak through mutant GLUT1.
\end{abstract}

\section{Introduction}

Paroxysmal dyskinesias (PDs) are characterized by involuntary movements triggered by certain stimuli such as sudden movement or prolonged exercise. They are classified into paroxysmal kinesigenic dyskinesia (PKD or PKC for paroxysmal kinesigenic choreoathetosis), paroxysmal nonkinesigenic dyskinesia (PNKD), and paroxysmal exertion-induced (PED) subtypes. PKD, the most common form, typically presents with brief (seconds to minutes) and frequent dyskinetic attacks provoked by sudden movements, responding well to anticonvulsive agents such as carbamazepine. Attacks in PNKD last from 10 minutes up to several hours and can be triggered by caffeine, alcohol, or fatigue. Response to treatment is usually poor. The most successful agents are benzodiazepines, and some benefit has been reported for flunarizine and

Nonstandard abbreviations used: $\left[\mathrm{Ca}^{2+}\right]_{i}$, free cytosolic $\mathrm{Ca}^{2+}$ concentration; CSF, cerebrospinal fluid; FDG-PET, $\left[{ }^{18} \mathrm{~F}\right]$ fluorodeoxy-glucose positron emission tomography; $\mathrm{PD}$, paroxysmal dyskinesia; $\mathrm{PED}$, paroxysmal exertion-induced dyskinesia; PNKD, paroxysmal nonkinesigenic dyskinesia.

Conflict of interest: The authors have declared that no conflict of interest exists. Citation for this article: J. Clin. Invest. 118:2157-2168 (2008). doi:10.1172/JCI34438. carbamazepine. PED is characterized by dyskinesias induced by prolonged exercise of 15-60 minutes duration. The attacks last between 5 minutes and 2 hours and are typically restricted to the exercised limbs. Treatment is difficult, but some beneficial effects have been described for anticonvulsants. The dyskinesias in all of these 3 syndromes include varying combinations of dystonic, choreatic, athetotic, and ballistic features (1). Rare syndromes of PDs combined with other symptoms have also been described, for example, paroxysmal choreoathetosis/spasticity with episodic ataxia (CSE) (2), benign familial infantile convulsions and paroxysmal choreoathetosis (ICCA) (3), and autosomal recessive rolandic epilepsy with PED and writer's cramp (RE-PED-WC) (4). PDs can occur as either inherited or sporadic/symptomatic forms. For some symptomatic cases, lesions within the thalamus or the basal ganglia have been described (5).

Although mutations in the MR1 gene located on chromosome 2 q35 encoding a protein with unknown function have been described for PNKD $(6,7)$, the underlying pathophysiology of PDs remains largely elusive. Analogous to idiopathic epilepsies and other episodic neurological disorders $(8,9)$, disruption of ionic homeostasis by dysfunction of channels or transporters 
could play a pathophysiological role in PDs. This hypothesis was recently confirmed by detection of a mutation in a calcium-activated potassium channel gene in a family with idiopathic epilepsy and a form of PD most resembling PNKD (10). Linkage to chromosome 16p12-q12 has been described for PKD, ICCA, benign familial infantile seizures alone (BFIS), and RE-PED-WC $(3,4,11)$, but an underlying genetic defect has not been identified for any of these disorders. The genetics and pathophysiology of PED are currently not known.

Passive diffusion of glucose across the blood-brain barrier and into red blood cells is facilitated by the glucose transporter 1 (GLUT1). Heterozygous mutations occurring mainly de novo in the SLC2A1 gene encoding GLUT1 impair glucose transport into the brain by a haploinsufficiency mechanism (12-14). The classic phenotype of this condition, designated as GLUT1 deficiency syndrome (MIM606777), comprises deceleration of head growth, severe motor and mental developmental delay, epilepsy, and further complex neurological symptoms with spasticity, dystonia, and ataxia. Hypoglycorrhachia, a lowered ratio of the glucose levels in cerebrospinal fluid (CSF) and serum, is the clinical laboratory hallmark. Treatment with a ketogenic diet that provides ketone bodies as alternative energy source for the brain is reported to result in marked improvement of seizures and other neurological symptoms. Since the first description of GLUT1 deficiency syndrome in 1991 (12), a carbohydrate-responsive phenotype, with clinical features aggravated by fasting and improved after carbohydrate intake (15), and a single patient with mild mental retardation and intermittent ataxia (16) or predominant dystonia (17), but without epilepsy, have been reported. PDs and hematological symptoms have not been described to be associated with SLC2A1 mutations so far, although GLUT1 is the primary glucose transporter of red blood cells.

Here we present the details of a neuro-hematological syndrome in a 3-generation family, in which PED is associated with epilepsy, mild developmental delay, and hemolytic anemia with deformed erythrocytes. We identified the underlying genetic defect - a unique mutation predicting a 4-amino acid deletion in the pore region of GLUT1 - and have elucidated its molecular pathophysiology - a cation leak with reduced glucose transport - using a range of electrophysiological, transport, and biochemical assays. Two additional mutations in the same gene were subsequently found in independent families with PED associated with epilepsy or migraine, but without hemolysis or echinocytosis. Altogether, we believe we have identified mutations in GLUT1 as a first genetic cause of PED and propose that a unique cation leak of this glucose transporter can induce hematological symptoms.

\section{Results}

\section{Clinical evaluation of family PED1}

Individual III-2 (40 years of age, index case). With onset at 6 years, the index patient (see Figure 1A for kindred) has been suffering from episodes of involuntary exertion-induced dystonic, choreoathetotic, and ballistic movements lasting minutes to hours (usually 5-15 minutes). The attacks occur after prolonged exercise, affecting exclusively the exercised limbs (Supplemental Movie 1; supplemental material available online with this article; doi:10.1172/JCI34438DS1). After puberty, the symptoms became more frequent. He had to stop to work as a boatman and started a professional retraining program at age 32 . The interictal neurological examination was normal. Neuropsychological evaluation revealed slight deficits in attention concerning complex tasks and verbal memory.

After about 20 minutes of continuous exercise at 150 watts with a heart rate of about 120 beats per minute on a bicycle ergometer (below anaerobic level), dystonia and involuntary choreoathetotic and ballistic movements occurred reproducibly in the legs (Supplemental Movie 1). The dyskinesias usually disappeared immediately when exercise was stopped but sometimes continued while at rest for up to 2 hours after exercising. Serum lactate and pyruvate and a lactate-ischemia test of the forearm revealed normal values confirming normal metabolism in skeletal muscle. Ictal and interictal EEG recordings were normal, as were electromyography, nerve conduction studies, somato-sensoryevoked potentials and central conduction times as determined using transcranial magnetic stimulation.

Treatment with carboanhydrase inhibitors (acetazolamide, up to $250 \mathrm{mg}$ /day, and diclofenamide, $150 \mathrm{mg}$ /day) showed limited benefit for 6-9 months. Low doses of levodopa (50-100 mg/day) and gabapentin (up to $4,800 \mathrm{mg} /$ day) had no effect. A ketogenic diet was started and its effects were monitored using a bicycle ergometer with constant efforts of 150 watts. Before and within the first 4 days of the diet, dyskinesias occurred 17-21 minutes after onset of exercising. At days 5 to 7 , dyskinesias appeared only after 40 minutes. After 4 weeks of the diet, the patient had to stop the ergometer test after 50 minutes due to exhaustion, but no dyskinesias appeared. However, the patient stopped the ketogenic diet despite its effectiveness due to difficulties in following the strict composition of the dietary regime.

Individual II-2 (60 years of age). The mother of the index patient reported very similar but less severe symptoms since school age ("cramps" and similar involuntary movements after exercise). Beside the typical symptoms of the lower extremities, she also reported stiffness of her fingers after typing. In contrast to the disease course of her son, there were less frequent symptoms since the age of 35 and they disappeared completely after the age of 45 . At the age of 53, the neurological examination was normal. Only mild deficits were found during neuropsychological assessment, affecting verbal memory and frontal functions. One hour of continuous exercise on a bicycle ergometer did not provoke dyskinesias. EEG was unremarkable.

Individual IV-1 ( 15 years of age). The first son of the index patient showed laboratory features of hemolytic anemia immediately after birth and needed a single blood transfusion on his second day of life. Thereafter, his anemia remained stable with hemoglobin concentrations around $110 \mathrm{~g} / \mathrm{l}$. His motor development was overall normal; he walked without support at 15 months but showed some clumsiness and mild ataxia and dystonia. His mental development was mildly delayed. He attended a special school, and psychological testing at 8 years revealed a learning handicap. With onset at 16 months of age, he suffered from recurrent short epileptic seizures with myoclonic jerks, nodding, sudden loss of muscle tone, eye deviation, and disturbed consciousness. These seizures occurred predominantly in the morning before breakfast and improved after carbohydrate intake. In addition, he developed ongoing exertion-induced dystonia of the legs at the age of 4 years.

Neurological examination at 7 years of age revealed mild gait ataxia and a disturbance of fine motor skills. Neuropsychological assessment showed an attention deficit and normal intelligence. Body weight, height, and head circumference were normal. EEG showed multiple generalized irregular spike-wave discharges, espe- 
$A_{\text {I }}$

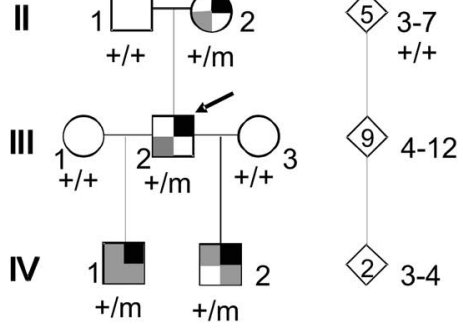

( $)$ hemolytic anemia

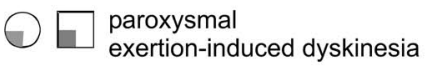

$\bigcirc \square$ epilepsy

(1) developmental delay

(n) \# unaffected
B
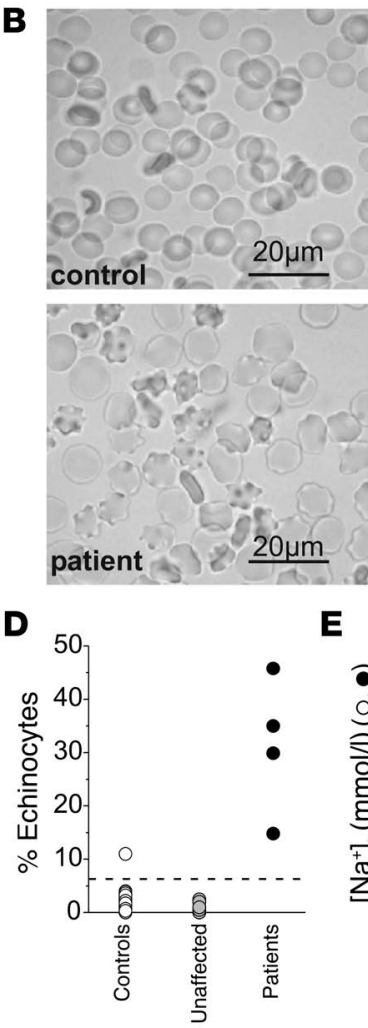

E
C
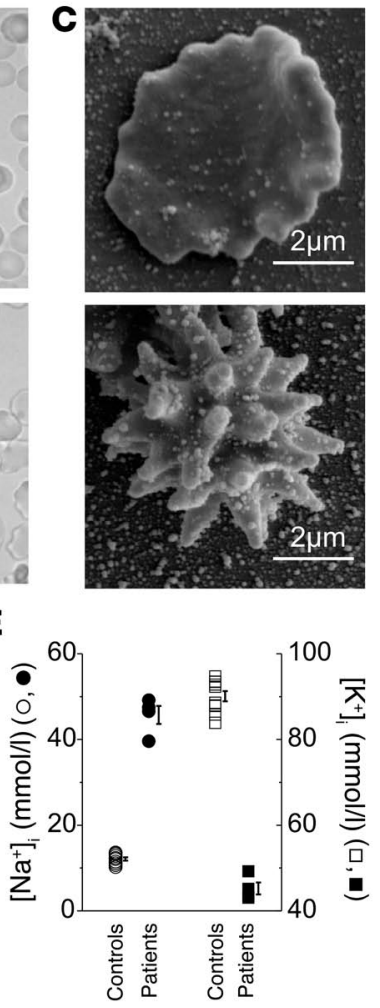

\section{Figure 1}

Clinical data of family PED1. (A) Pedigree with clinical and genetic status. $+/ \mathrm{m}$, individuals carrying the Q282_S285del mutation (see Figure 2); +/+, individuals with 2 WT alleles of SLC2A1. An arrow marks the index patient. (B) Wet blood smears from a normal control and the index patient. Scale bar: $20 \mu \mathrm{m}$. (C) Electron microscopy showing 2 states of echinocytes from the index patient. Scale bar: $2 \mu \mathrm{m}$. (D) Quantitative analysis of echinocytes from 132 controls, 11 unaffected family members, and the 4 patients. All echinocyte counts from patients were above the normal value (6.3\%, dashed line). (E) Compared with 10 controls, $\left[\mathrm{Na}^{+}\right]_{\mathrm{i}}$ was increased and $\left[\mathrm{K}^{+}\right]_{\mathrm{i}}$ decreased in erythrocytes from patients $\left(P<1 \times 10^{-10}\right)$. Individual values for patients and mean \pm SEM are shown (D and $\mathbf{E}$ ). cially in the morning and after fasting, accompanied by atonic and myoclonic seizures and atypical absences. Seizures were stopped and the EEG normalized after intravenous infusion of glucose. Treatment with antiepileptic drugs, including clonazepam, sultiam, clobazam, and lamotrigine was initiated in his second year of life and reduced seizure frequency. A ketogenic diet was started at 11 years of age; it has lead to complete seizure control and a marked improvement of his performance in school.

Individual IV-2 (9 years of age). The second son of the index patient is a half brother of IV-1. Marked neonatal icterus prompted blood tests leading to the diagnosis of hemolytic anemia. A transfusion was performed at 2 weeks of age, but further transfusions were not necessary. He walked at 12 months, and spoke his first words at 12 months. Subsequently, clumsiness, mild coordination disturbances, and mild delay in his mental development were apparent. However, he attended regular kindergarten and school. With onset at 3 years of age, he had irregularly recurrent seizures with lethargy, loss of muscle tone, and erratic eye movements for a duration of about 10 to 15 minutes. EEG showed mild slowing of background activity and bursts of focal and generalized irregular spike-waves with frontal predominance. Continuous video-EEG monitoring revealed these spike-waves occurred mainly in the early morning. Body weight and height and head circumference were at the 75th, 50th, and 25 th percentile, respectively. Examination using the HAWIK-III revealed verbal, performance, and full scale IQ of 82, 75, and 77, respectively, at 7 years of age. A ketogenic diet resulted in complete resolution of seizures and improvement of his mental development.

Further clinical and laboratory studies. All 4 affected family members presented with intermittent scleral icterus, splenomegaly, a macrocytic hemolytic anemia with reticulocytosis, decreased haptoglobin, and increased bilirubin (Supplemental Table 1). For those individu- als for whom follow-up investigations were available (IV-1, IV-2), there was a gradual increase of erythrocytic mean corpuscular volume with ageing. There was no evidence for a deficiency of erythrocytic enzymes, a hemoglobinopathy, an autoimmune anemia, a vitamin deficiency, or any other known cause of membrane defects (Supplemental Table 1). Using the method of Storch and coworkers (18), we found increased amounts of deformed red blood cells (acanthocytes/echinocytes), ranging from 14.8\% to 41.6\% (normally $<6.3 \%$ ) of all erythrocytes in wet blood smears of isotonically diluted blood samples in affected family members (Figure 1, B-D). Electron microscopy demonstrated that these cells were echinocytes (Figure 1C). In erythrocytes of all affected individuals, $\left[\mathrm{Na}^{+}\right]_{\mathrm{i}}$ was increased and $\left[\mathrm{K}^{+}\right]_{\mathrm{i}}$ decreased (Figure $1 \mathrm{E}$ ). No evidence for a disturbance of cellular energy metabolism was found (energy load, according to ref. 19, was 0.92-0.93, normal range was 0.90-0.96; Supplemental Table 1). A McLeod phenotype was absent. A bone marrow puncture displayed elevated erythropoiesis in II-2.

Investigations of CSF revealed glucose levels at or below the lower limit of the normal range. The glucose CSF/serum ratio ranged from 0.39 to 0.55 and was mildly reduced compared with controls (0.62-0.68), but it was not as low as in most patients with GLUT1 deficiency syndrome (usually <0.40) (14). CSF lactate was also mildly reduced (Supplemental Table 1 ).

History, neurological, and hematological examinations were unremarkable for all other family members, including both parents and the 5 siblings of the index patient's mother, indicating a de novo mutation with a dominant trait in family PED1 (Figure 1, A and D).

Neuroimaging studies. Cranial MRI showed hypointense signals in $\mathrm{T} 2$ - and $\mathrm{T} 2 *$-weighted images (T2 and $\mathrm{T} 2 *$ indicate standard imaging sequences of the MRI; see Supplemental Methods) in the 


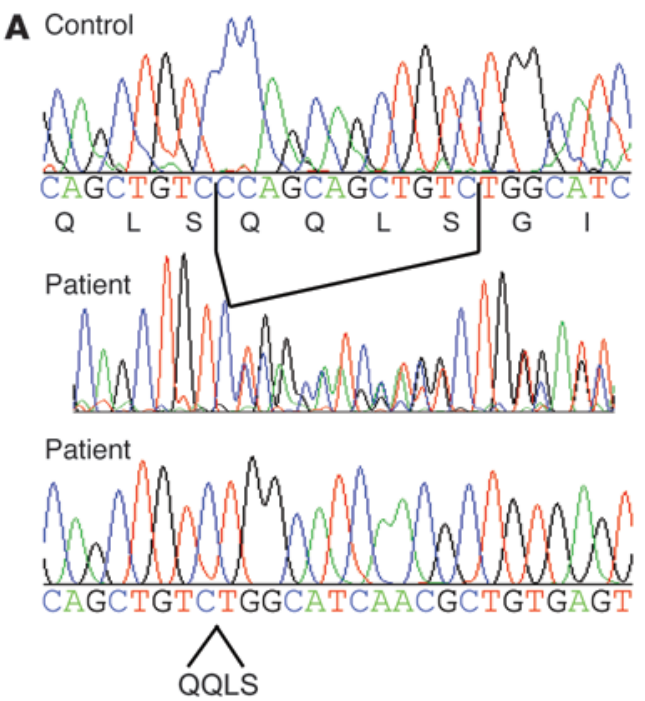

B

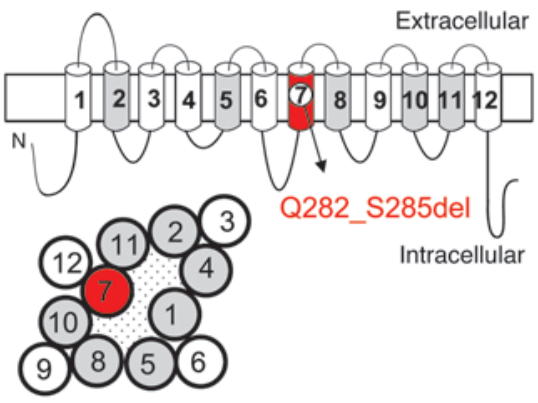

c
GlUT1-human
GIUT1-mouse
GlUT1-rat
GlUT1-drosophila
GlUT2-human
GLUT3-human
GLUT4-human
GLUT5-human
GLUT6-human
GLUT7-human
GLUT8-human

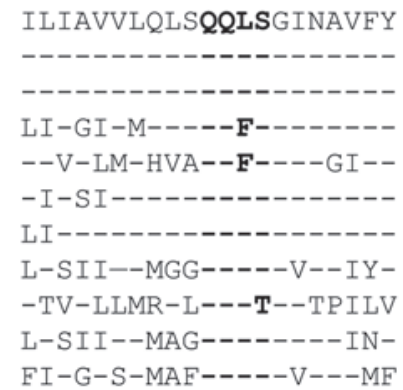

caudal putamen, which were more pronounced in II-2 as in III-2 (Supplemental Figure 1A). Such alterations are known as early MRI signs from patients with multiple system atrophy (20) but may occasionally be seen in healthy controls as well, in particular in older age groups. MRI scans of IV-1 and IV-2 were normal. Interictal positron emission tomography using $\left[{ }^{18} \mathrm{~F}\right]$ fluorodeoxy-glucose (FDG-PET) representing a measure of cerebral glucose metabolism revealed a decreased signal in the left more pronounced than in the right thalamus when compared with 17 age-matched controls using statistical parametric mapping (SPM99) (Supplemental Figure 1B). The significance of this finding could be confirmed when individuals II-2, III-2, and the index case of family PED5 (see below) were compared as a group with 17 controls (Supplemental Figure 1C), as the only difference which survived correction for multiple comparisons $(P=0.05)$ was found in the left thalamus. All 3 right-handed patients suffer from PED alone, without epilepsy or mental retardation. In addition, we

\section{Figure 2}

Genetic investigations. (A) Genomic sequences of a normal control (upper panel), the index patient (middle panel), and the cloned, mutant allele from the index patient (lower panel), revealing a 12-bp deletion at nucleotide 1,022 and loss of amino acids 282-285 (QQLS). The black line below the upper panel denotes the exact deleted region of the 12 bp. (B) Proposed structure of GLUT1 with 12 transmembrane segments (T1-12) and the location of Q282_S285del (red). A central pore (hatched region in the extracellular view) may be formed by segments marked in gray or red. (C) The deleted motif QQLS is highly conserved among species and other glucose transporters.

found a less significant hypermetabolism in the putamen bilaterally (Supplemental Figure 1D).

\section{Genetic investigations of family PED1}

The combination of neuro-hematological symptoms and reduced CSF glucose levels (a hallmark of GLUT1 deficiency syndrome) (12-14) prompted us to sequence SLC2A1 encoding GLUT1, the glucose transporter of both erythrocytes and the blood-brain barrier in a candidate gene approach. We identified an in frame 12-bp deletion (c.1022_1033del) in exon 6 predicting the loss of the 4 amino acids QQLS (p.Q282_S285del) within the seventh transmembrane segment (T7). T7 is assumed to constitute a highly conserved, central part of the pore-forming region involved in glucose specificity of the transporter (Figure 2, A and B) $(21,22)$. The deletion cosegregated with the disease status (Figure 1A) and was excluded in 150 normal controls, in both parents of II-2 and further nonaffected family members (see Figure 1A). The parental status of I- 1 was genetically proved, confirming a de novo mutation in II-2. To this end, we tested 12 highly polymorphic microsatellite markers from 12 different chromosomes (D1S518, D2S1777, D4S1652, D4S1009, D8S1128, D10S677, D12S391, D14S608, D16S539, D18S851, D20S604, D22S689) using genomic DNA of the probands I-1, I-2, II-2, and II-3. Clear paternity and maternity of I-1 and I-2, respectively, for II- 2 and II-3 was confirmed.

\section{Functional studies of WT and mutant Q282_S285del GLUT1 transporters}

To prove its functional relevance, we introduced c.1022_1033del into the cDNA of GLUT1 and expressed WT and mutant transporters in Xenopus oocytes. Equal amounts of cRNA of either the WT or mutant gene were injected and all experiments were conducted in parallel to avoid any bias between WT and mutant transporters (see Supplemental Methods). We first measured the uptake of 3-O-methyl-D-glucose using a previously published protocol (15). Glucose uptake was significantly reduced for Q282_S285del compared with WT transporters (Figure 3A). When we determined the glucose uptake rate in erythrocytes from patients in comparison with the one from normal controls, we found a significant reduction for the patients $(P<0.05$; Figure 3B). As expected, the difference between patients and controls was less pronounced than between mutant and WT transporters in oocytes, since the patients carry 1 WT allele encoding normally functioning transporters, which are expressed in their erythrocytes.

Since the ionic changes observed in erythrocytes could be directly induced by the mutant transporter, we performed electrophysiological experiments in oocytes. The resting membrane potential was depolarized (Q282_S285del, $-19 \pm 2 \mathrm{mV}$; WT, $\left.-43 \pm 2 \mathrm{mV} ; \mathrm{H}_{2} \mathrm{O},-39 \pm 4 \mathrm{mV} ; n=10 ; P<0.0001\right)$, and the input 
A
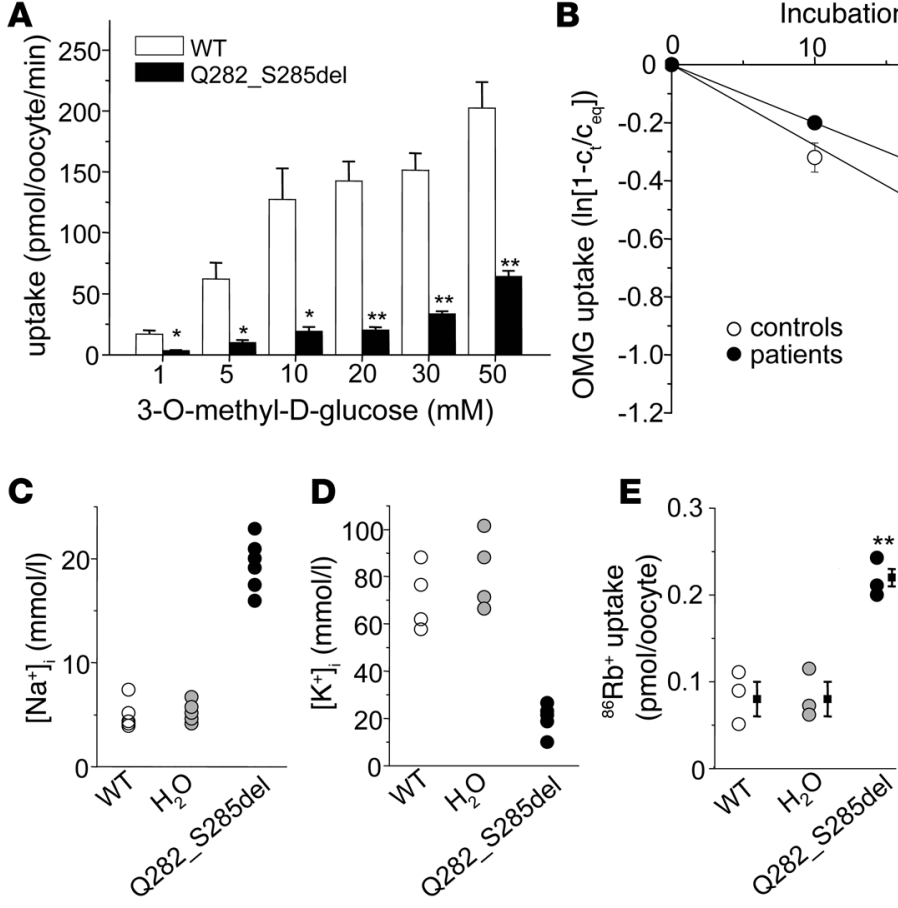

Figure 3

Functional studies. (A) Reduced glucose uptake recorded in oocytes injected with mutant compared with WT cRNA. Shown are representative results recorded from $3 \times 10$ oocytes for each data point. ${ }^{*} P<0.05,{ }^{*} P<0.01$. (B) Glucose uptake as determined in 4 charges of erythrocytes from 3 patients of family PED1 carrying the Q282_S285del mutation (III-2, IV-1, IV-2; the index patient III-2 was measured twice and both of his sons once each) and in 10 charges from different normal controls. Plotted is the logarithm $\ln \left(1-c_{t} / c_{e q}\right)$, with $c_{t}$ being the assimilated radioactivity after time $t$ and $c_{e q}$ the 1 in the equilibrium (after $25 \mathrm{~min}$ ), versus time yielding a linear function. The mean slope values of the linear fits in controls were $-0.0280 \pm 0.0018 \mathrm{~s}^{-1}$ and in patients $-0.0201 \pm 0.0006 \mathrm{~s}^{-1}(P<0.05)$. Error bars are smaller than symbol size for the patients. ( $\mathbf{C}$ and D) Using ion-selective electrodes, $\left[\mathrm{Na}^{+}\right]_{i}$ and $\left[\mathrm{K}^{+}\right]_{i}$ were recorded in oocytes, injected as indicated. $\left[\mathrm{Na}^{+}\right]_{i}$ was increased and $\left[\mathrm{K}^{+}\right]_{i}$ decreased for mutant compared with WT transporters. $n=6$ for each group; $P<1 \times 10^{-6}$ (mutant versus WT), $P<1 \times 10^{-7}$ (mutant versus $\left.\mathrm{H}_{2} \mathrm{O}\right)($ C) $; n=4-5$, $P<0.001$ (mutant versus both WT and $\mathrm{H}_{2} \mathrm{O}$ ) (D). $P$ values were calculated based on the potential differences between the ion-selective electrode and the conventional electrode. Therefore, error bars are not shown for the calculated ionic concentrations (see Supplemental Methods). (E) ${ }^{86} \mathrm{Rb}^{+}$flux experiments in oocytes show increased values for the mutation compared with the WT. Shown are values from $3 \times 8$ oocytes for each condition. ${ }^{* *} P<0.01$. (F) Increased ${ }^{86} \mathrm{Rb}$ f flux was also demonstrated in erythrocytes from 3 patients (III-2, IV-1, IV-2) compared with erythrocytes from 11 normal controls. ${ }^{* *} P<0.001$. Representative experiments from individual batches of oocytes are shown (A and $\mathbf{C}-\mathbf{E})$. Individual values $(\mathbf{C}-\mathbf{F})$ and mean \pm SEM (error bars in $\mathbf{A}, \mathbf{B}$, $\mathbf{E}$, and F) are presented.

resistance was significantly decreased (Q282_S285del, $0.14 \pm 0.04$ $\mathrm{M} \Omega$; WT, $\left.1.60 \pm 0.26 \mathrm{M} \Omega ; \mathrm{H}_{2} \mathrm{O}, 1.93 \pm 0.31 \mathrm{M} \Omega ; n=10 ; P<0.0001\right)$ for oocytes injected with mutant cRNA compared with those injected with WT cRNA or water, as revealed by 2 -microelectrode voltage clamping. We used ion-selective electrodes (ISEs) to record electrolytes in oocytes in the presence of ouabain and bumetanide, which block cation transfer by other transporters (see Methods). Oocytes injected with mutant cRNA had significantly increased $\left[\mathrm{Na}^{+}\right]_{\mathrm{i}}$ and decreased $\left[\mathrm{K}^{+}\right]_{\mathrm{i}}$ compared with those injected with WT cRNA or $\mathrm{H}_{2} \mathrm{O}$ (Figure 3 , C and D). Next, we performed ${ }^{86} \mathrm{Rb}^{+}$flux measurements in the presence of ouabain and bumetanide to confirm a potassium leak in a direct dynamic experiment. As expected, ${ }^{86} \mathrm{Rb}^{+}$uptake was significantly increased for oocytes injected with mutant cRNA compared with those injected with WT cRNA or

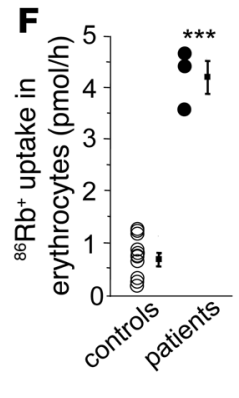

$\mathrm{H}_{2} \mathrm{O}$ (Figure 3E). In erythrocytes from 3 of the 4 patients, ${ }^{86} \mathrm{Rb}^{+}$flux was about 6-fold increased compared with 11 normal controls (Figure 3F). All these differences occurred in the absence of glucose, suggesting a permanent monovalent cation leak of the mutant transporter, independent of the glucose transport function. This was confirmed by incubating erythrocytes from patients and controls with 0 or $15 \mathrm{mM}$ glucose for 2 hours, which did not influence the changes in intracellular electrolyte concentrations (see Supplemental Table 2).

To further characterize the leak conductance induced by the mutation, we performed additional electrophysiological experiments with native erythrocytes from 3 patients of family PED 1 and 3 normal controls. In accordance with our results in oocytes, the whole-cell conductance was significantly increased in the patients' erythrocytes (controls, $83 \pm 16$ $\mathrm{pS} ; n=4$; patients, $363 \pm 12 \mathrm{pS} ; n=6 \mathrm{pS}$; $P<0.05$; Figure $4, \mathrm{~A}-\mathrm{D})$. Very stable recording conditions allowed multiple solution exchanges, revealing a permeability of the mutant transporter for $\mathrm{K}^{+}>\mathrm{Na}^{+}>\mathrm{Ca}^{2+}>$ $\mathrm{N}$-methyl-D-glucamine ${ }^{+}\left(\mathrm{NMDG}^{+}\right)$, as the reversal potential was shifted increasingly in the hyperpolarizing direction, and the whole-cell inward currents decreased in this order (Figure 4D). To confirm the increased $\mathrm{Ca}^{2+}$ permeability of the mutant transporter, we determined the erythrocyte free cytosolic $\mathrm{Ca}^{2+}$ concentration $\left(\left[\mathrm{Ca}^{2+}\right]_{\mathrm{i}}\right)$ by flow cytometry using fluo 3 as a $\mathrm{Ca}^{2+}$-sensitive dye. Under steady-state conditions, patients' erythrocytes exhibited a significantly higher fluo3 fluorescence intensity than those of controls $(7.6 \pm 0.5$ vs. $6.0 \pm 0.3$ relative fluorescence units; $n=18-19 ; P<0.02$; Figure 4E), indicating an increased $\left[\mathrm{Ca}^{2+}\right]_{\mathrm{i}}$. Applying a $\mathrm{Ca}^{2+}$ depletion/repletion protocol (Figure 4, $\mathrm{F}$ and $\mathrm{G}$ ) revealed a significantly faster reincrease in fluo3 fluorescence intensity in patients' erythrocytes, suggestive of a higher $\mathrm{Ca}^{2+}$ leak conferred by the mutant transporter.

Increased $\left[\mathrm{Ca}^{2+}\right]_{\mathrm{i}}$ triggers suicidal erythrocyte death and we hypothesized that erythrocyte suicide could underlie the anemia seen in our patients (23). We therefore tested for death markers such as erythrocyte shrinkage, breakdown of the phospholipid asymmetry, activation of the protease calpain, and hemolysis in blood from controls and patients incubated in vitro for 24 hours at $37^{\circ} \mathrm{C}$. Upon incubation in $\mathrm{Ca}^{2+}$-containing, but not in $\mathrm{Ca}^{2+}$-free, medium erythrocytes from patients exhibited a lower cell size/ volume, indicating $\mathrm{Ca}^{2+}$-induced shrinkage (Supplemental Figure $2 \mathrm{~A}$ ). In addition, phospholipid asymmetry of the erythrocyte membrane was broken down in a higher percentage of erythrocytes from patients than of control cells. Breakdown of the phos- 
A Control
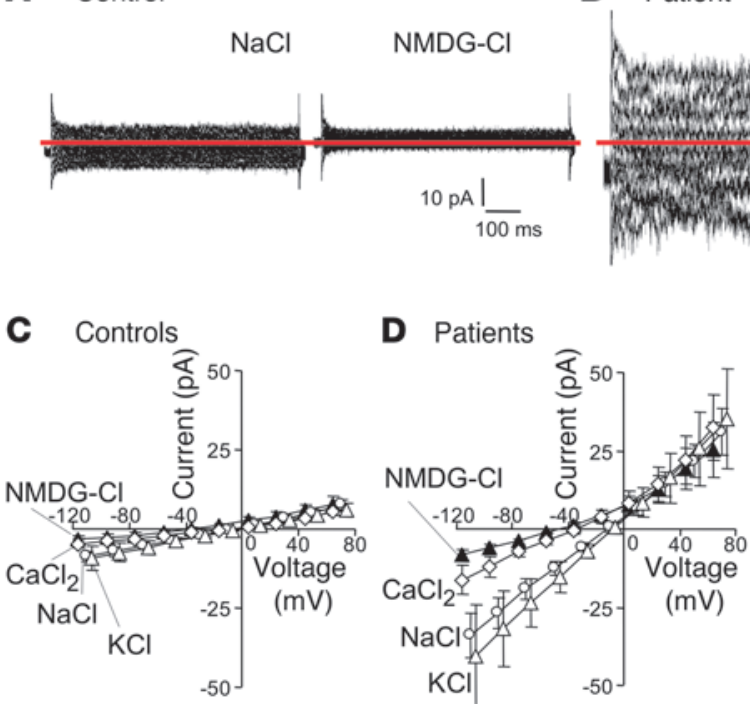

E Steady state $\mathrm{Ca}^{2+}$

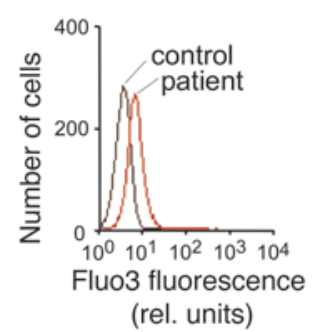

\footnotetext{
$\mathbf{F}$
}
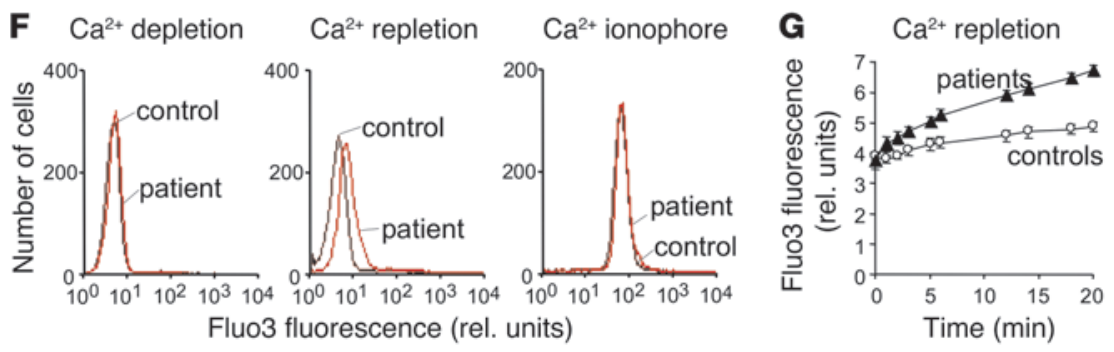

Figure 4

Functional analysis of the cation leak in erythrocytes from patients of family PED1. (A and B) Representative whole-cell current traces recorded from erythrocytes of a control (A) and a patient (B) with Na-gluconate in the pipette and $\mathrm{NaCl}$ in the bath solution (left panel) and after isoosmotic replacement of $\mathrm{NaCl}$ by $\mathrm{N}$-methyl-D-glucamine-chloride (NMDG-Cl) in the bath (right panel). (C and D) Mean current-voltage (I-V) relationships ( \pm SEM) for control (C) $(n=3-4)$ and patients' erythrocytes (D) $(n=5-6)$ recorded as in $\mathbf{A}$ and $\mathbf{B}$ with $\mathrm{NaCl}$ bath solution (open circles) and after isoosmotic replacement of $\mathrm{NaCl}$ by NMDG-Cl (closed triangles), $\mathrm{CaCl}_{2}$ (open diamonds), or $\mathrm{KCl}$ (open triangles) in the bath. In patients' erythrocytes (D), the reversal potential of the I-V curve shifted by $-30 \pm 4,-20 \pm 2$, and $+11 \pm 2 \mathrm{mV}$ (mean $\pm \mathrm{SEM} ; n=5-6$ ) from that recorded in $\mathrm{NaCl}$ solution following substitution with $\mathrm{NMDG}-\mathrm{Cl}, \mathrm{CaCl}_{2}$, and $\mathrm{KCl}$, respectively. (E) Histogram recorded by flow cytometry in erythrocytes from controls (black line) and patients (red line) incubated in $\mathrm{Ca}^{2+}$-containing $\mathrm{NaCl}$ solution, depicting the fluo3 fluorescence intensity as a measure of the steady-state intracellular $\left(\left[\mathrm{Ca}^{2+}\right]_{\mathrm{i}}\right)$. $(\mathbf{F}$ and $\mathbf{G})$ Histograms $(\mathbf{F})$ and time course (G) of changes in fluo3 fluorescence intensity of control (black line in $\mathbf{F}$; open circles in $\mathbf{G}$ ) and patients' erythrocytes (red line in $\mathbf{F}$; close triangles in $\mathbf{G}$ ) following $\mathrm{Ca}^{2+}$ depletion by incubation for 30 minutes in $\mathrm{Ca}^{2+}$-free $\mathrm{NaCl}$ solution ( $\mathbf{F}$, left panel, and $\mathbf{G}, 0$-minute values) and $\mathrm{Ca}^{2+}$ repletion $(\mathbf{F}$, middle panel, and $\mathbf{G})$. As a positive control experiment, the histogram in ( $\mathbf{F}$, right panel) shows the fluo3 fluorescence of $\mathrm{Ca}^{2+-}$-permeabilized erythrocytes from controls (black line) and patients (red line) indicating equal fluo3 dye loading of both cell populations under these conditions (mean values \pm SEM, $49 \pm 8$ and $55 \pm 8$ relative fluorescence units in erythrocytes from controls and patients, respectively; $n=4$ ). The data in $\mathbf{G}$ are averaged geometrical means ( \pm SEM; $n=14-16)$ of the fluorescence distribution. Lines represent exponential fits yielding the time constant of $\left[\mathrm{Ca}^{2+}\right]_{\text {i }}$ repletion (controls, $\tau=16.3 \pm 3.4 \mathrm{~min}$; patients, $\tau=7.1 \pm 1.6 \mathrm{~min}$; $n=14-16, P<0.05 ;$ 2-tailed Welch-corrected $t$ test).

pholipid asymmetry was assessed in flow cytometry by annexin $\mathrm{V}$ binding to phosphatidylserine exposed at the outer membrane face (Supplemental Figure 2, B and C). Moreover, patients' erythrocytes, but not those of controls, activated the $\mathrm{Ca}^{2+}$-dependent endopeptidase calpain as indicated in immunoblot by cleavage of the inactive p 80 proenzyme into its active forms p78 and p76 (Supplemental Figure 2D). Finally, a higher percentage of erythrocytes from patients hemolyzed as compared with those of controls (Supplemental Figure $2 \mathrm{E})$. Taken together, the data clearly indicate that erythrocytes from patients of family PED1 are more prone to undergo suicidal death than those from controls.

On a molecular basis, the cation leak could be explained by the structural importance of the highly conserved QQLS motif, that may form a bottle neck within the transporter's pore (22), which is deleted in mutant Q282_S285del transporters (see Discussion).

\section{Further clinical, genetic, and functional investigations in other families with PED} As the hyperkinesias in PED occur exclusively after prolonged periods of exercise, i.e., under conditions of an increased energy demand, it is pathophysiologically reasonable that a reduced glucose transport across the blood-brain barrier can cause such symptoms. To investigate whether this could be a general principle for PED, we sequenced the SLC2A1 gene encoding GLUT1 in 4 additional families with PED (named PED2-5). The clinical features of 3 of these families, with 6 or 4 members affected by PED in 3 generations, each have been previously published.

In family PED2 (ref. 24; Figure 5A), typical childhood onset PED is combined with epilepsy with absences or complex partial seizures, mild learning disabilities, and an irritable behavior with increased impulsivity in 6 affected members.

In family PED3, childhood onset PED is combined with epilepsy (absences, complex partial or generalized tonic-tonic seizures) and mild developmental delay. Hyperactivity and migraine with visual auras was noticed in 1 of the 4 affected individuals (25).

In family PED4, juvenile onset PED was originally described to be combined with minor permanent dystonia, postural tremor and chorea (barely noticed by the patients themselves), and migraine without aura (26). Recent reexamination of this family revealed 2 important differences with regard to the original description: (a) individual III-8 in the meantime has developed attacks of dystonia precipitated by exercise and (b) individual III-14 presents with a phenotype that is entirely different from other family members. She has a persistent, fixed abnormal posture affecting the right foot, associated with some discomfort in the right foot and leg. General neurological examination is normal apart from the presence of some "give-way" weakness of the 

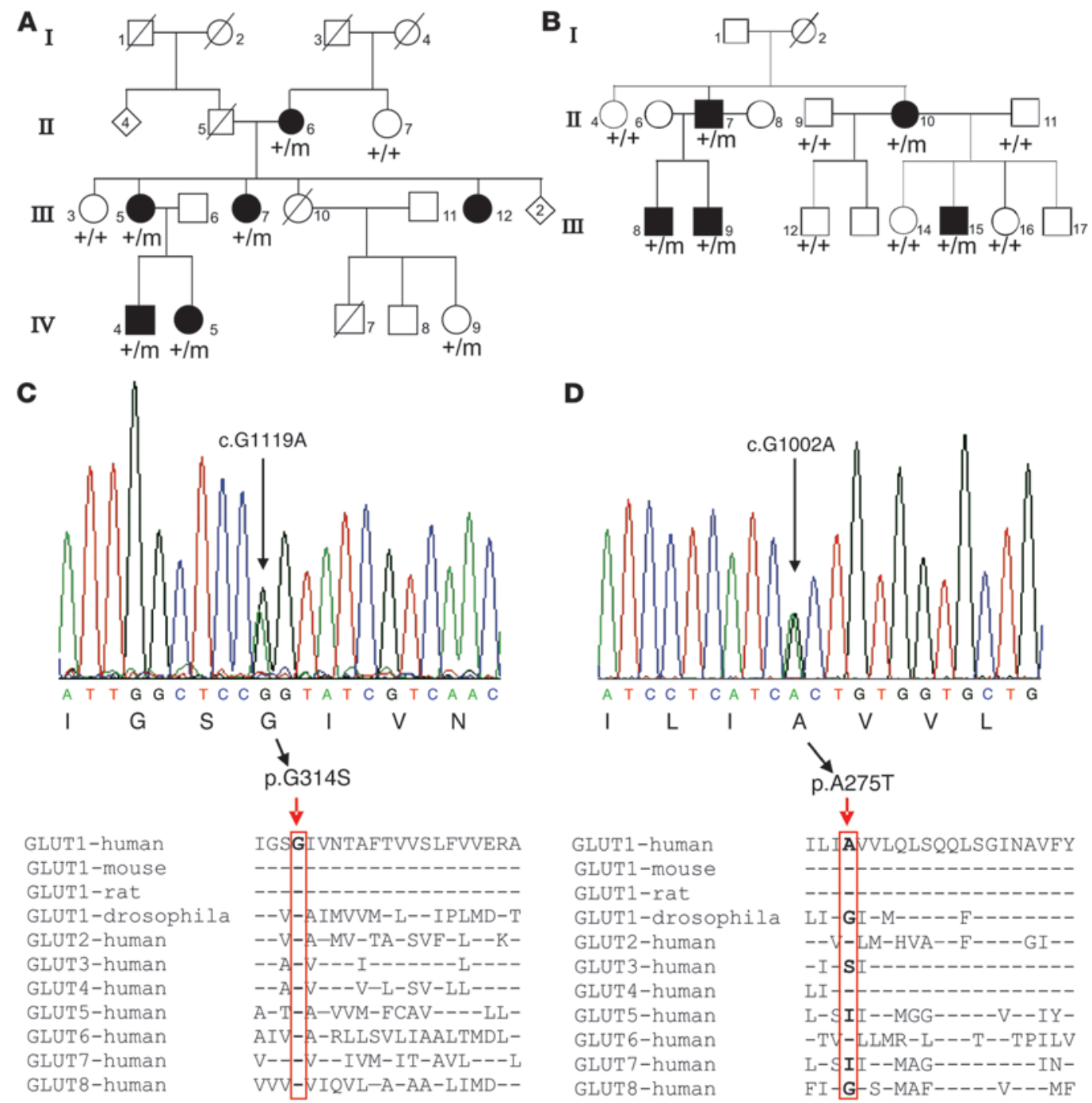

D
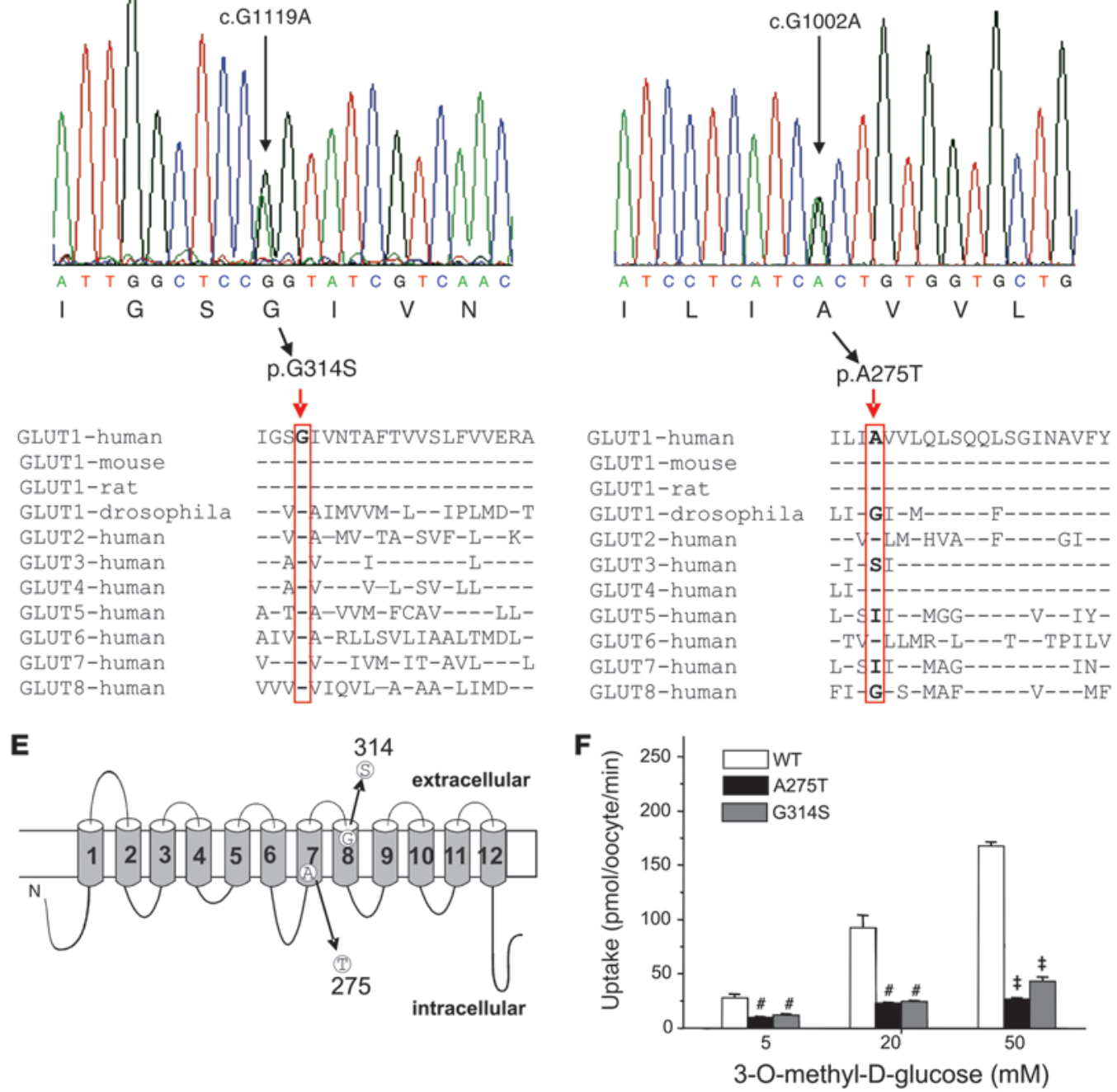

\section{Figure 5}

Genetic and functional analysis of further families with PED. (A) Partial pedigree of family PED2 (see ref. 24 for full pedigree). Individuals affected by PED, epilepsy, mild mental retardation, and impulsivity are shown as filled symbols, and open symbols represent unaffected individuals. Symbols with diagonal lines represent deceased individuals. (B) Pedigree of family PED4 (modified after ref. 26; see text). Filled symbols denote individuals affected by PED. +/+ denotes 2 WT alleles, whereas $+/ m$ denotes heterozygous mutation carriers. (C and D) DNA sequences shown for patients III-5 from family PED2 and II-7 from PED4 reveal point mutations c.G1119A (C) and c.G1002A (D), predicting the substitutions p.G314S and p.A275T, respectively. Lower panels show G314 is highly, while A275 is a bit less, conserved among species and other glucose transporters. (E) Localization of the 2 novel mutations in transmembrane domains 7 and 8 of GLUT1. (F) Glucose uptake in oocytes was reduced for both mutations (shown are representative results recorded from 1 batch of $3 \times 10$ oocytes for each glucose concentration, mean \pm SEM; $\left.{ }^{\#} P<0.01,{ }^{\ddagger} P<0.001\right)$. 
A

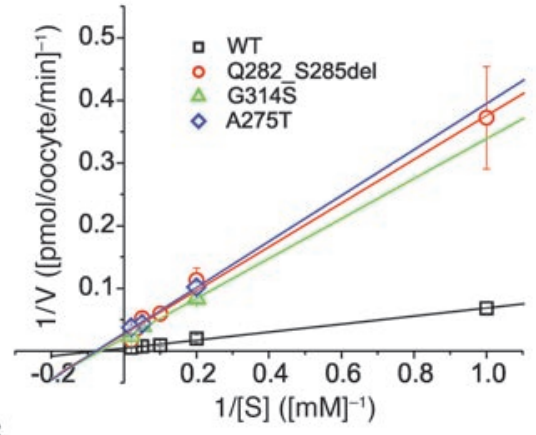

C

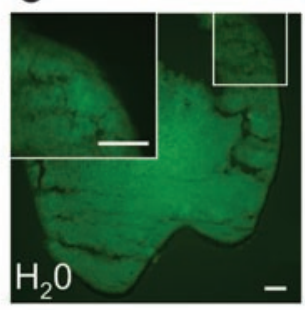

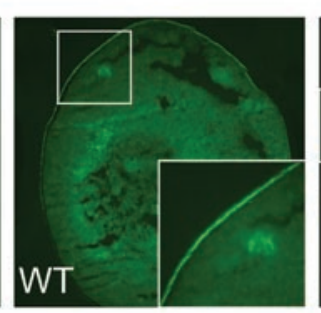
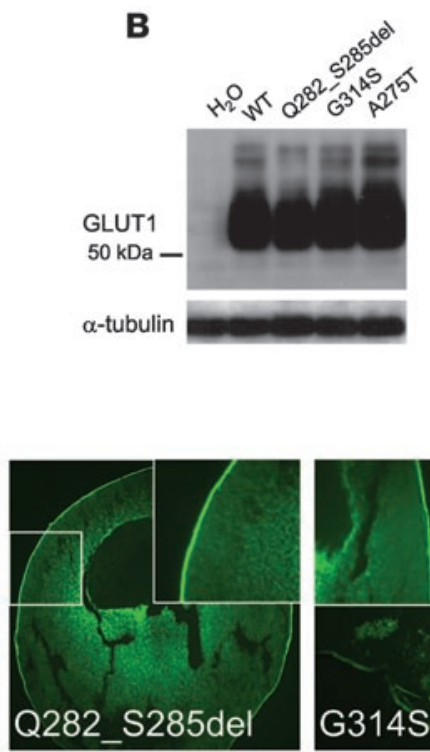
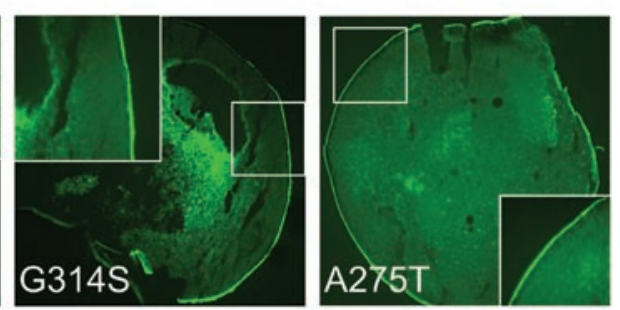

\section{Figure 6}

Kinetics, protein stability, and trafficking of all 3 mutants compared with WT GLUT1 transporters. (A) Kinetic analysis of glucose uptakes in oocytes (as shown in Figure $3 A$ and Figure 5F) according to Lineweaver-Burk. Lines represent linear fits to the data points using the equation $1 / \mathrm{V}$ $(1 /[S])=1 / V_{\max }+K_{m} / V_{\max } \times 1 /[S]$, with [S] being the concentration of the substrate OMG and $V$ being the uptake velocity in pmol/oocyte/min. The $y$-axis intercept equals $1 / V_{\max }$ and the $x$-axis intercept represents $-1 / K_{m}$ (see Supplemental Methods). $V_{\max }$ was markedly reduced for all 3 mutations compared with the WT without obvious effects on $K_{m}$. The following values were obtained: WT, $V_{\max }=213 \pm 35 \mathrm{pmol} / \mathrm{oocyte} / \mathrm{min}$ and $K_{m}=$ $13.7 \pm 2.5 \mathrm{mM}$; Q282_S285del, $V_{\max }=37 \pm 8 \mathrm{pmol} /$ oocyte $/ \mathrm{min}$ and $K_{m}=13.0 \pm 3.2 \mathrm{mM}$; G314S, $V_{\max }=49 \pm 11$ and $K_{m}=15.5 \pm 4.9 \mathrm{mM}$; A275T, $V_{\max }=35 \pm 4 \mathrm{pmol} /$ oocyte/min and $K_{m}=13.0 \pm 2.3 \mathrm{mM}$. (B) Western blots obtained from oocytes injected with equal amounts of cRNA showed a similar amount of protein for all mutations and the WT, but no respective band for oocytes injected with $\mathrm{H}_{2} \mathrm{O}$ as a negative control; $\alpha$-tubulin was used as a loading control. (C) Immunocytochemical analysis of injected oocytes using an anti-GLUT1 antibody revealed similar stainings of the surface membranes for all 4 clones, suggesting a normal trafficking of the mutant proteins to the surface membrane. Scale bars: $100 \mu \mathrm{m}$.

arms and legs. She has no paroxysmal symptoms, and her symptoms are not worsened by exertion. Her symptoms are very much in keeping with the syndrome of "fixed dystonia" (27). Many patients with fixed dystonia are felt to have a psychogenic origin for their symptoms, and indeed, psychogenic dystonia has previously been reported in family members of patients with organic genetic dystonia (28). Given these differences to the original description, we here present a modified pedigree with PED as the only unequivocal and consistent phenotype in this family (Figure 5B).

Family PED5, with 4 affected individuals in 2 generations, has not been described before. The index case and her sister have typical childhood-onset PED alone, affecting mainly the legs after 15-120 minutes of walking. Both the daughter and the nephew of the index case (son of the sister affected by PED) mainly have a moderate developmental delay. In addition, the daughter has some minor ataxia and the nephew has epilepsy with complex partial seizures. Neither of these individuals have experienced attacks reminiscent of $\mathrm{PED}$.

Laboratory investigations did not reveal signs of hemolytic anemia in the other families (i.e., normal values for hemoglobin ( $\mathrm{Hb}$ ), mean corpuscular volume, haptoglobin, bilirubin, reticulocytes, and all other tested hematologic and standard serum parameters), with the exception of a consistent mild normocytic and normochrome anemia (Hb between 11 and $12 \mathrm{~g} / \mathrm{dl}$, normal values for haptoglobin, reticulocyte count, bilirubin, transferrin, ferritin, and $\mathrm{Fe}$ ) in all 4 affected members of family PED3, and an $\mathrm{Hb}$ of 11.5 $\mathrm{g} / \mathrm{dl}$ in individual IV-5 of PED2 (other values were normal, Hb was normal in other affected family members). There was no increase in the percentage of echinocytes in families PED3 and PED5, as examined by the method of Storch et al. (18), and intraerythrocytic electrolytes were normal in 3 affected members of family PED3, III-15 of PED4, and all affected members of PED5. The CSF/serum glucose ratios were obtained from 3 affected members of family PED3 (0.35, 0.45, and 0.49), III-15 of PED4 (0.54), and the index case of PED5 (0.50).

Direct sequencing of SLC $2 A 1$ revealed 2 different point mutations in families PED2 and PED4, which were found in all individuals affected by PED. In family PED2, we detected a c.G1119A mutation, predicting substitution of serine for glycine at position 314 (p.G314S), located in the eighth transmembrane segment (Figure $5, \mathrm{C}$ and $\mathrm{E}$ ). The mutation was also detected in a nonaffected individual and must have been transmitted by her deceased mother, who was also reported to be unaffected, indicating most probably 2 cases of nonpenetrance. One affected individual (III-12) did not agree to participate in the study. In family PED4, a c.G1002A mutation was identified, predicting substitution of threonine for alanine at position 275 (p.A275T) at the cytoplasmic end of transmembrane segment 7 (Figure 5, D and E). This mutation perfectly cosegregates with the PED phenotype. G314 is highly and A275 less well conserved in GLUT1 from other species and other human glucose transporters (Figure 5, C and D). Both mutations were not detected in 150 normal controls. No mutations in SLC2A1 could be detected in families PED 3 and PED5 by sequencing the entire coding region and adjacent splice sites. Multiplex ligation-dependent probe ampli- 
fication (MLPA) analysis, performed for all exons of SLC2A1, also excluded an abnormal copy number variation in these 2 families.

To demonstrate that these 2 mutations lead to a decreased glucose transport as well, we introduced them into the cDNA of SLC2A1 and measured glucose uptake in Xenopus oocytes. The uptake was largely reduced for both mutations compared with the WT, confirming their pathophysiological significance (Figure 5F). ${ }^{86} \mathrm{Rb}^{+}$uptake was normal for both mutations (G314S, $0.35 \pm 0.01$ pmol/oocyte; A275T, $0.22 \pm 0.06 \mathrm{pmol} /$ oocyte; WT, $0.28 \pm 0.06$ pmol/oocyte; $\mathrm{H}_{2} \mathrm{O}, 0.16 \pm 0.01 \mathrm{pmol} /$ oocyte), suggesting that these 2 mutations may not induce an increased permeability of GLUT1 to cations as was observed for the Q282_S285del mutation.

Kinetics, protein stability, and trafficking of mutant and WT GLUT1 transporters. A decreased glucose transport rate, as determined for all 3 GLUT1 mutants, could be due to several different defects on the protein level, such as a decrease in (a) the transport rate of the mutated transporter itself; (b) affinity of the transporter to glucose; (c) protein stability; or (d) protein trafficking to the surface membrane. To differentiate among these different mechanisms, we performed additional data evaluation and experiments. A kinetic analysis of the uptake measurements in oocytes shown in Figure 3A and Figure 5F revealed that all mutations markedly decreased the $V_{\max }$ without affecting the $K_{m}$ (Figure 6A). Western blots suggested an equal production and stability of the mutants compared with the WT protein (Figure 6B), and immunocytochemistry indicated that all 3 mutants were properly inserted in the cell-surface membrane (Figure 6C). We therefore conclude, that the mutant transporters have lost their intrinsic ability to transport glucose across the cell membrane without affecting $\mathrm{K}_{\mathrm{m}}$, protein stability or trafficking.

\section{Discussion}

Our genetic and pathophysiological investigations establish SLC2A1 as the first gene to our knowledge for PED and strongly support the hypothesis that an energy deficit upon exertion caused by a reduced glucose transport rate is a major cause of this paroxysmal movement disorder. GLUT1 is the primary molecule to mediate glucose transport (a) into erythrocytes, (b) across the endothelium of the blood-brain barrier, and (c) into and out of astrocytes (29). The latter 2 sites of transport might both contribute to the observed neurological symptoms, since they are both involved in the delivery of glucose to nerve cells. It is conceivable that the energy demand increases under conditions of prolonged exercise and exceeds the energy supply, which is reduced in PED patients with GLUT1 mutations, as shown by our uptake experiments and by previous functional studies of the mutated sites $(30,31)$. This hypothesis is supported by the therapeutic success of both intravenously administered glucose during exercise and a permanent ketogenic diet, which changes the main energy source of the brain from glucose to ketone bodies, as observed in the index patient of family PED1 and his older son. The absence of GLUT1 mutations in 2 families with a similar clinical phenotype suggests the involvement of other genes in the pathogenesis of PED, although we did not exclude mutations in the promoter or other untranslated regions of SLC2A1 in this study, which theoretically, might be the cause of the disease in these families.

Since the basal ganglia are particularly sensitive to hypoxia and energy deficits and are key players in the generation of dyskinesias $(32,33)$, the clinically relevant energy deficit in PED patients might be localized in the basal ganglia. This hypothesis is supported by the mild permanent abnormalities in the basal ganglia or the thala- mus, as a putative projection area, which were detected in MRI and FDG-PET (Supplemental Figure 1). The pronounced hypometabolism in the left versus the right thalamus could be explained by the right handedness of all 3 PED patients investigated with FDG-PET. The clinical observation that PEDs very specifically occur within the exercised limbs furthermore suggests that the energy deficit only occurs in a relatively small number of neurons, which are crucial for the coordination of the activated muscle groups.

The normal hematological parameters in families PED2 and PED4, combined with the fact that the 2 mutations identified in these families did not reveal an increased cation permeability of GLUT1, indicate that the cation leak is not a necessary condition to produce symptoms of PED. Patients with other GLUT1 defects are characterized by severe developmental delay, epilepsy, ataxia, and spasticity (GLUT1 deficiency syndrome), reminiscent of the milder phenotype of individual IV-1 in family PED1, but PDs or hematological symptoms have not yet been reported as part of this syndrome (14). Also, PDs have not been described in other disorders presenting with various neurological symptoms and deformed erythrocytes (the so-called neuroacanthocytoses) (34). Using the same methods in 5 patients from 3 families with GLUT1 deficiency syndrome and different SLC2A1 mutations, we observed normal erythrocyte morphology and intracellular electrolytes.

The cation leak is thus unique for the mutation Q282_S285del identified in family PED1, in which all affected family members suffered from a consistent hemolytic anemia with echinocytosis. Furthermore, our analysis revealed a normal energy metabolism of erythrocytes from patients of family PED1, supporting the hypothesis that the defective glucose transport is not responsible for the anemia and altered erythrocyte morphology. Instead, our detailed experiments in oocytes and erythrocytes revealed a definite increase in $\mathrm{K}^{+}, \mathrm{Na}^{+}$, and $\mathrm{Ca}^{2+}$ permeability, which can explain the altered erythrocyte lifetime. Erythrocytes with an increased cation leak are prone to swell and eventually hemolyze osmotically. We hypothesize that the $\mathrm{Ca}^{2+}$-triggered suicidal death - as observed in erythrocytes from patients of family PED1 - delays the hemolysis by transiently shrinking the cells, leading to echinocyte formation. In addition, suicidal death-associated phosphatidylserine exposure acts as an "eat-me" signal and promotes the recognition and engulfment of the dying erythrocytes by macrophages, thus clearing the cell prior to its disastrous hemolysis (23). In the present work, pronounced suicidal death of the patient erythrocytes resulted in their increased hemolysis (see Supplemental Figure 2E) due to the absence of phagocytes in vitro. Hemolysis may also occur in vivo, if erythrocyte death rates exceed the clearance capacity of the phagocytic system.

Genetic defects of the anion exchanger of the erythrocyte membrane (AE1, band 3 protein) cause familial hemolytic anemia with stomatocytosis by inducing a pathological cation leak of AE1 (35, 36). Also in analogy to this well defined syndrome, our findings suggest that the observed cation leak of GLUT1 is responsible for the hematological abnormalities found in family PED1. Our results hence constitute the second genetic defect in which idiopathic hemolytic anemia with an altered morphology of red blood cells is associated with a cation leak of their membrane and provide a further proof of principle for the link between those 2 conditions.

On a molecular level, the cation leak of the mutated GLUT1 molecule can be explained by the specific location of the 4-amino acid deletion. The Q282_S285del mutation is the first one reported to our knowledge in transmembrane segment 7 , which probably plays a crucial role in the transporter's pore $(21,22)$. Moreover, 
the deleted amino acids QQLS are hypothesized to form a bottle neck within the pore of GLUT1 (22). Molecular modelling using the published coordinates of a GLUT1 model (22) suggest a widening of the pore region (central channel) by the Q282_S285del deletion, which can explain the observed permanent cation leak (Supplemental Figure 3).

\section{Methods}

Patients. All patients and their unaffected relatives (or their legal representatives) gave written informed consent to participate in the study. All affected and most of the unaffected members of the families PED1-5 were personally interviewed and examined by experienced neurologists or pediatricians; for some of the unaffected individuals, histories were reported to be unremarkable by first degree relatives. Individuals with a normal neurological and hematological history, neurological examination, analysis of erythrocyte morphology as far as it was available (see below) were considered as unaffected. All procedures were in accordance with the Helsinki Convention and were approved by the Ethical Committees of the University of Ulm, the University of Tübingen, the University of Bari, the National Hospital for Neurology and Neurosurgery, and the Institute of Neurology Joint Research. The clinical features of families PED2-4 have been described previously (24-26).

Analysis of erythrocyte morphology. All procedures were performed as described previously (18). Fresh blood samples $(5 \mathrm{ml})$ were immediately mixed with the same amount of isotonic $\mathrm{NaCl}$ solution containing 10 units of heparin per ml. After 1-2 hours, 1 drop of the mixture was mounted between an object slide and a glass cover slip and examined by phasecontrast light microscopy with $\times 1,000$ magnification (oil immersion). Five hundred erythrocytes were counted, and the proportion of deformed cells was calculated. All red blood cells with spicules corresponding to type AI/ AII acanthocytes and echinocytes in Redman's classification (37-39) were considered to be deformed (18) and classified as echinocytes as revealed by scanning electron microscopy (39). The amounts of echinocytes are expressed as percent of total erythrocytes. Using this method, the normal range of echinocytes is less than $6.3 \%$ of all erythrocytes (99th percentile of 132 healthy and disease controls), with a specificity of 0.98 and a sensitivity for detecting echinocytosis/acanthocytosis in genetically confirmed choreo-acanthocytosis of 1.0 (18). This morphological analysis was performed in families PED1, PED3, and PED5. Fresh blood samples were not available for families PED2 and PED4.

For scanning electron microscopy, the samples were prepared in the same way as described above for light microscopy and then were fixed by adding $2.5 \%$ glutaraldehyde. Dehydration was performed using graded alcohols and a critical point dryer (Bal-Tec). The samples were shadowed by platinum (Bal-Tec) and examined in a scanning electron microscope (DSM 962; Carl-Zeiss) at $10 \mathrm{kV}$.

Determination of intracellular ionic concentrations in erythrocytes. EDTA blood samples from patients and normal controls were drawn on the same day and shipped to our laboratory overnight. Several milliliters of blood were centrifuged for 10 minutes at 2,000 g. Erythrocytes were washed 3 times with $10 \mathrm{ml}$ of choline chloride $(150 \mathrm{mM})$, centrifuged after each washing step, and the supernatant was discarded. Washed erythrocytes were lysed by 3 alternate steps of freezing in liquid nitrogen and defrosting in a water bath at $80^{\circ} \mathrm{C}$, followed by centrifugation for 1 hour at 3,500 $\mathrm{g}$. The supernatant was transferred in $1.5-\mathrm{ml}$ Eppendorf vials and centrifuged again for 1 hour at 5,000 g. Aliquots of the supernatant were analyzed with a Dimension RxL chemistry analyzer (Dade Behring) using the urine program to determine $\left[\mathrm{Na}^{+}\right]_{\mathrm{i}}$ and $\left[\mathrm{K}^{+}\right]_{\mathrm{i}}$ in erythrocytes.

To evaluate the effect of external glucose on $\left[\mathrm{Na}^{+}\right]_{i}$ and $\left[\mathrm{K}^{+}\right]_{i}$, the serum was removed and exchanged against an $\mathrm{Ca}$-free electrolyte solution con- taining 0 or $15 \mathrm{mM}$ glucose $\left(138 \mathrm{mM} \mathrm{NaCl}, 2 \mathrm{mM} \mathrm{CaCl}_{2}, 5.4 \mathrm{mM} \mathrm{KCl}\right.$, $1 \mathrm{mM} \mathrm{MgCl}_{2}$, and $10 \mathrm{mM}$ HEPES, pH 7.4). After 2 hours on a shaker, we proceeded with washing steps as described above.

Genetic analysis, mutagenesis and RNA preparation, oocyte preparation and injection, uptake experiments in erythrocytes and oocytes using radioactively labelled glucose and ${ }^{86} \mathrm{Rb}^{+}$, western blots, and immunocytochemistry were performed by using standard procedures, which are described in Supplemental Methods.

Determination of $\left[\mathrm{Na}^{+}\right]_{i}$ and $\left[\mathrm{K}^{+}\right]_{i}$ in oocytes. Oocytes injected with equal amounts of cRNA for WT or mutant GLUT1 transporters, or with water as a control, were kept overnight in modified Barth's solution. Specific blockers of the $\mathrm{Na}^{+} / \mathrm{K}^{+}$ATPase and the $\mathrm{Na}^{+} / \mathrm{K}^{+} / 2 \mathrm{Cl}^{-}$cotransporter, $0.5 \mathrm{mM}$ ouabain and $5 \mu \mathrm{M}$ bumetanide, respectively, were added on the following day. Recordings were performed in parallel for mutant and WT transporters as well as for water-injected oocytes on day 3 after injection. $\left[\mathrm{Na}^{+}\right]_{i}$ and $\left[\mathrm{K}^{+}\right]_{i}$ were determined using 1 ion-selective and 1 conventional microelectrode, which were pulled from borosilicate glass capillaries (150TF-10; Science Products) using a DMZ-Universal Puller (Zeitz). Conventional electrodes were filled with $3 \mathrm{M} \mathrm{KCl}$ and had a final resistance of less than $1 \mathrm{M} \Omega$. ISEs had tip openings of about $1 \mu \mathrm{m}$ and were prepared by drying for at least 15 minutes at $200^{\circ} \mathrm{C}$ and subsequent silanizing with $20 \mu \mathrm{N}$-(Trimethylsilyl) dimethylamine (TMSDMA; Fluka 41720) in a glass bottle $(250 \mathrm{ml})$ at the same temperature. They were filled with either $\mathrm{Na}^{+}$Ionophore I - Cocktail A (Fluka 71176) or $\mathrm{K}^{+}$Ionophore I - Cocktail B (Fluka 60398). After removing air bubbles, $100 \mathrm{mM} \mathrm{NaCl}$ (Na-ISE) or $10 \mathrm{mM} \mathrm{KCl} \mathrm{(K-ISE)} \mathrm{was} \mathrm{added.} \mathrm{ISEs}$ were calibrated using solutions with different $\mathrm{NaCl}$ and $\mathrm{KCl}$ concentrations, with the sum of both ion concentrations being constant at $150 \mathrm{mM}$. Calibration curves were generated by fitting the obtained data points with a Nikolsky-Eisenmann function (further details see Supplemental Methods). Recordings from 1 batch of oocytes injected with WT, mutant cRNAs, or water were done in parallel, i.e., for each recording, oocytes were taken in a randomized manner from those 3 groups. Initial recordings were done in a blinded fashion, but after recording from the first 3 oocytes, it was already obvious which group contained the oocytes injected with mutant cRNA due to the large differences. For statistical analysis, $P$ values were not calculated for the different ion concentrations but for the differences between the potentials of both electrodes (ISE and conventional electrode) for mutant versus WT and mutant versus $\mathrm{H}_{2} \mathrm{O}$, since the potential differences are normally distributed while the calculated ion concentrations are not due to the nonlinear transformation, according to the Nikolsky-Eisenmann equation (see Supplemental Methods). Data were analyzed using Microsoft Excel (Microsoft) and Origin (Microcal Software) software.

Patch-clamp recordings and determination of $\left[\mathrm{Ca}^{2+}\right]_{i}$ in erythrocytes. Standard whole-cell patch clamping was performed at room temperature using borosilicate glass pipettes (10-12 M $\Omega$ pipette resistance, GC150 TF-10; Harvard Apparatus). Currents were sampled at $10 \mathrm{kHz}$ and low-pass filtered at $3 \mathrm{kHz}$ using an EPC-9 amplifier, Pulse software (HEKA), and an ITC-16 Interface (Instrutech). Liquid junction potentials were estimated and corrected according to Barry and Lynch (40). A relative mobility of $1.0388,0.3000,0.2000,0.2400,1.0000,0.6820,0.3300,0.4880$, and 0.3610 was assumed for $\mathrm{Cl}^{-}$, HEPES - , ATP, EGTA, $\mathrm{K}^{+}, \mathrm{Na}^{+}, \mathrm{NMDG}^{+}, \mathrm{Ca}^{2+}$, and $\mathrm{Mg}^{2+}$, respectively. Whole-cell currents were evoked by voltage steps from a $-30 \mathrm{mV}$ holding potential to voltages between $-100 \mathrm{mV}$ and $+80 \mathrm{mV}$ and digitally low-pass filtered at $2 \mathrm{kHz}$. Current values were analyzed by averaging the whole-cell currents between 100 and $600 \mathrm{~ms}$ of each square pulse. The bathing solution contained $125 \mathrm{mM} \mathrm{NaCl}, 32 \mathrm{mM}$ HEPES, $5 \mathrm{mM} \mathrm{KCl}$, $5 \mathrm{mM}$-glucose, $1 \mathrm{mM} \mathrm{MgSO}_{4}$, and $1 \mathrm{mM} \mathrm{CaCl}_{2}$ titrated with $\mathrm{NaOH}$ to $\mathrm{pH}$ 7.4. The pipette solution contained $115 \mathrm{mM}$ Na-D-gluconate, $10 \mathrm{mM}$ HEPES, $5 \mathrm{mM} \mathrm{MgCl}_{2}, 1 \mathrm{mM} \mathrm{Na}$-ATP, and $1 \mathrm{mM}$ EGTA titrated with $\mathrm{NaOH}$ to $\mathrm{pH}$ 7.4. The $\mathrm{NaCl}$-based bathing solution was replaced during continu- 
ous whole-cell recording by a NMDG-Cl solution (180 mM NMDG, 10 mM HEPES, $1 \mathrm{mM} \mathrm{MgCl}_{2}$, and $1 \mathrm{mM} \mathrm{CaCl}_{2}$ titrated with $\mathrm{HCl}$ to $\mathrm{pH} 7.4$ ), a $\mathrm{CaCl}_{2}$ solution (100 $\mathrm{mM} \mathrm{CaCl}_{2}, 10 \mathrm{mM} \mathrm{HEPES}$, and $1 \mathrm{mM} \mathrm{MgCl}_{2}$ titrated with $\mathrm{CaOH}$ to $\mathrm{pH}$ 7.4), or a $\mathrm{KCl}$ solution (150 mM KCl, $10 \mathrm{mM}$ HEPES, $1 \mathrm{mM} \mathrm{CaCl}_{2}$, and $1 \mathrm{mM} \mathrm{MgCl}_{2}$ titrated with $\mathrm{KOH}$ to $\mathrm{pH}$ 7.4).

To determine $\left[\mathrm{Ca}^{2+}\right]_{i}$ in the steady state, erythrocytes were washed and loaded for 20 minutes at $37^{\circ} \mathrm{C}$ with the $\mathrm{Ca}^{2+}$-sensitive dye, fluo3 $\mathrm{AM}$ ( $4 \mu \mathrm{M}$ final concentration; Calbiochem), washed again twice, and finally suspended in $\mathrm{CaCl}_{2}$-containing $\mathrm{NaCl}$ solution (see patch-clamp experiments). For the $\mathrm{Ca}^{2+}$ depletion/repletion protocol, cells were washed, loaded with $4 \mu \mathrm{M}$ fluo3 $\mathrm{AM}\left(20\right.$ minutes at $\left.37^{\circ} \mathrm{C}\right)$, and washed again in $\mathrm{Ca}^{2+}$-free $\mathrm{NaCl}$ solution (125 mM NaCl, $32 \mathrm{mM}$ HEPES, $5 \mathrm{mM} \mathrm{KCl}, 5 \mathrm{mM}$ D-glucose, and $1 \mathrm{mM} \mathrm{MgSO}_{4}$ titrated with $\mathrm{NaOH}$ to $\mathrm{pH}$ 7.4). One aliquot of the cells was resuspended in $\mathrm{Ca}^{2+}$-free $\mathrm{NaCl}$ solution (to determine the 0 minute value of the $\mathrm{Ca}^{2+}$ reuptake), another in $\mathrm{Ca}^{2+}$ containing $\mathrm{NaCl}$-solution. Steady-state $\left[\mathrm{Ca}^{2+}\right]_{\mathrm{i}}$ and time-dependent reincrease of $\left[\mathrm{Ca}^{2+}\right]_{\mathrm{i}}$ during $\mathrm{Ca}^{2+}$ repletion was assessed by flow cytometry (FACSCalibur; Becton Dickinson). The fluo3 fluorescence intensity was determined in fluorescence channel FL-1 with an excitation wavelength of $488 \mathrm{~nm}$ and an emission wavelength of $530 \mathrm{~nm}$. To control for equal dye loading, fluo3 fluorescence was determined following $\mathrm{Ca}^{2+}$ permeabilization $(1 \mu \mathrm{M}$ ionomycin in $\mathrm{Ca}^{2+}$-containing $\mathrm{NaCl}$ solution). During $\mathrm{Ca}^{2+}$ repletion, the increase in the geometrical mean of the fluorescence intensity was fit exponentially $\left(y=y_{\max }-\left(y_{\max }-y_{0}\right) e^{(-t / \tau)}\right.$; with $t$ being the time of repletion, $\tau$ the time constant, $y_{0}$ the fluorescence intensity at time 0 minutes, and $y_{\max }$ the maximal fluorescence intensity after $\mathrm{Ca}^{2+}$ reuptake).

Further experiments with erythrocytes (breakdown of the phospholipid asymmetry and cell volume, calpain activation, and hemolysis) are described in Supplemental Methods.

Statistics. Two-tailed, unpaired Student's $t$ tests were used for statistical analysis, if not indicated otherwise, with $P$ values as indicated in the text or figure legends. Data are shown as mean \pm SEM or as individual values.

The procedures for brain imaging (MRI and FDG-PET) and molecular modelling are described in Supplemental Methods.

\section{Acknowledgments}

We thank all family members who participated in this study; H.-J. Christen, B. Walter, T. Perniola, P. Ventura, M. Niemann, A. Hermann, and M. Andres for following some of the examined fam- ily members or providing additional clinical information; Mike Mueckler for providing the cDNA of SLC2A1 (GLUT1); P. Walther for performing the scanning electron microscopy; D. Gläser for performing MLPA; J. Liebrich and A. Bellan-Koch for technical assistance; and A.C. Ludolph, S. Schorge, M. Walker, and M. Bommer for helpful discussion. This study was supported by grants from the Bundesministerium für Bildung und Forschung (BMBF/NGFN2, 01GS0478 to H. Lerche; Genetic Network for Hereditary Movement Disorders [GeNeMove] 01GM0304 to T. Gasser), the European Union (Epicure, LSH 037315, to H. Lerche), and the DFG (Le1030/9-1, Le1030/10-1 to H. Lerche and Hu781/4-3 to S. Huber). Y.G. Weber and T.V. Wuttke were supported by a fellowship from the University of Ulm. H. Lerche is a Heisenberg fellow of the DFG.

Received for publication November 6, 2007, and accepted in revised form March 19, 2008.

Address correspondence to: Holger Lerche, Neurologische Klinik und Institut für Angewandte Physiologie, Universität Ulm, Zentrum Klinische Forschung, Helmholtzstr. 8/1, 89081 Ulm, Germany. Phone: 49-731-500-63117 or 49-731-177-5203; Fax: 49731-177-1202; E-mail: holger.lerche@uni-ulm.de.

Arnulf Pekrun's present address is: Prof.-Hess Pädiatrische Klinik, Bremen, Germany.

Eduard Kraft's present address is: Physikalische Medizin, Ludwig-Maximilians-Universität, Klinikum Grosshadern, Munich, Germany.

Felix M. Mottaghy's present address is: Department of Nuclear Medicine, University of Leuven, Leuven, Belgium.

Alexander Münchau's present address is: Klinik und Poliklinik für Neurologie, Universitätsklinikum Hamburg-Eppendorf, Hamburg, Germany.

Yvonne G. Weber, Alexander Storch, and Thomas V. Wuttke contributed equally to this work.
1. Bhatia, K.P. 2001. Familial (idiopathic) paroxysmal dyskinesias: an update. Semin. Neurol. 21:69-74.

2. Auburger, G., et al. 1996. A gene for autosomal dominant paroxysmal choreoathetosis/spasticity (CSE) maps to the vicinity of a potassium channel gene cluster on chromosome $1 \mathrm{p}$, probably within $2 \mathrm{cM}$ between D1S443 and D1S197. Genomics. 31:90-94.

3. Szepetowski, P., et al. 1997. Familial infantile convulsions and paroxysmal choreoathetosis: a new neurological syndrome linked to the pericentromeric region of human chromosome 16. Am. J. Hum. Genet. 61:889-898.

4. Guerrini, R., et al. 1999. Autosomal recessive rolandic epilepsy with paroxysmal exercise-induced dystonia and writer's cramp: delineation of the syndrome and gene mapping to chromosome 16p12-11.2. Ann. Neurol. 45:344-352.

5. Lee, M.S., and Marsden, C.D. 1994. Movement disorders following lesions of the thalamus or subthalamic region. Mov. Disord. 9:493-507.

6. Rainier, S., et al. 2004. Myofibrillogenesis regulator 1 gene mutations cause paroxysmal dystonic choreoathetosis. Arch. Neurol. 61:1025-1029.

7. Lee, H.Y., et al. 2004. The gene for paroxysmal nonkinesigenic dyskinesia encodes an enzyme in a stress response pathway. Hum. Mol. Genet. 13:3161-3170.
8. Lerche, H., Weber, Y.G., Jurkat-Rott, K., and Lehmann-Horn, F. 2005. Ion channel defects in idiopathic epilepsies. Curr. Pharm. Des. 11:2737-2752.

9. Cannon, S.C. 2006. Pathomechanisms in channelopathies of skeletal muscle and brain. Annu. Rev. Neurosci. 29:387-415.

10. Du, W., et al. 2005. Calcium-sensitive potassium channelopathy in human epilepsy and paroxysmal movement disorder. Nat. Genet. 37:733-738.

11. Weber, Y.G., et al. 2004. Benign familial infantile convulsions: linkage to chromosome $16 \mathrm{p} 12$-q12 in 14 families. Epilepsia. 45:601-609.

12. De Vivo, D.C., et al. 1991. Defective glucose transport across the blood-brain barrier as a cause of persistent hypoglycorrhachia, seizures, and developmental delay. N. Engl. J. Med. 325:703-709.

13. Seidner, G., et al. 1998. GLUT-1 deficiency syndrome caused by haploinsufficiency of the bloodbrain barrier hexose carrier. Nat. Genet. 18:188-191.

14. Wang, D., et al. 2005. Glut-1 deficiency syndrome: clinical, genetic, and therapeutic aspects. Ann. Newrol. 57:111-118.

15. Brockmann, K., et al. 2001. Autosomal dominant glut-1 deficiency syndrome and familial epilepsy. Ann. Neurol. 50:476-485.

16. Overweg-Plandsoen, W.C., et al. 2003. GLUT-1 deficiency without epilepsy-an exceptional case. J. Inherit. Metab. Dis. 26:559-563.

17. Friedman, J.R., et al. 2006. Atypical GLUT1 deficiency with prominent movement disorder responsive to ketogenic diet. Mov. Disord. 21:241-245.

18. Storch, A., Kornhass, M., and Schwarz, J. 2005. Testing for acanthocytosis A prospective readerblinded study in movement disorder patients. J. Neurol. 252:84-90.

19. Brown, G.C. 1992. Control of respiration and ATP synthesis in mammalian mitochondria and cells. Biochem. J. 284:1-13.

20. Kraft, E., Trenkwalder, C., and Auer, D.P. 2002. $\mathrm{T} 2 *$-weighted MRI differentiates multiple system atrophy from Parkinson's disease. Neurology. 59:1265-1267.

21. Hruz, P.W., and Mueckler, M.M. 2001. Structural analysis of the GLUT1 facilitative glucose transporter (review). Mol. Membr. Biol. 18:183-193.

22. Salas-Burgos, A., Iserovich, P., Zuniga, F., Vera, J.C., and Fischbarg, J. 2004. Predicting the three-dimensional structure of the human facilitative glucose transporter glut 1 by a novel evolutionary homology strategy: insights on the molecular mechanism of substrate migration, and binding sites for glucose and inhibitory molecules. Biophys. J. 87:2990-2999. 
23. Lang, K.S., et al. 2005. Mechanisms of suicidal erythrocyte death. Cell. Physiol. Biochem. 15:195-202.

24. Margari, L., et al. 2000. Familial paroxysmal exercise-induced dyskinesia and benign epilepsy: a clinical and neurophysiological study of an uncommon disorder. Neurol. Sci. 21:165-172.

25. Kamm, C., Mayer, P., Sharma, M., Niemann, G., and Gasser, T. 2007. New family with paroxysmal exercise-induced dystonia and epilepsy. Mov. Disord. 22:873-877.

26. Münchau, A., et al. 2000. A new family with paroxysmal exercise induced dystonia and migraine: a clinical and genetic study. J. Neurol. Neurosurg. Psychiatry. 68:609-614.

27. Schrag, A., Trimble, M., Quinn, N., and Bhatia, K. 2004. The syndrome of fixed dystonia: an evaluation of 103 patients. Brain. 127:2360-2372.

28. Bentivoglio, A.R., et al. 2002. Phenotypic variability of DYT1-PTD: does the clinical spectrum include psychogenic dystonia? Mov. Disord. 17:1058-1063.

29. Maher, F., Vannucci, S.J., and Simpson, I.A. 1994.
Glucose transporter proteins in brain. FASEB J. 8:1003-1011.

30. Mueckler, M., and Makepeace, C. 2004. Analysis of transmembrane segment 8 of the GLUT1 glucose transporter by cysteine-scanning mutagenesis and substituted cysteine accessibility. J. Biol. Chem. 279:10494-10499.

31. Hruz, P.W., and Mueckler, M.M. 1999. Cysteinescanning mutagenesis of transmembrane segment 7 of the GLUT1 glucose transporter. J. Biol. Chem. 274:36176-36180.

32. Pulsinelli, W.A. 1985. Selective neuronal vulnerability: morphological and molecular characteristics. Prog. Brain Res. 63:29-37.

33. DeLong, M.R., and Wichmann, T. 2007. Circuits and circuit disorders of the basal ganglia. Arch. Neurol. 64:20-24.

34. Danek, A., and Walker, R.H. 2005. Neuroacanthocytosis. Curr. Opin. Neurol. 18:386-392.

35. Bruce, L.J., et al. 2005. Monovalent cation leaks in human red cells caused by single amino-acid sub- stitutions in the transport domain of the band 3 chloride-bicarbonate exchanger, AE1. Nat. Genet. 37:1258-1263.

36. Guizouarn, H., Martial, S., Gabillat, N., and Borgese, F. 2007. Point mutations involved in red cell stomatocytosis convert the electroneutral anion exchanger 1 to a nonselective cation conductance. Blood. 110:2158-2165.

37. Feinberg, T.E., et al. 1991. Diagnostic tests for choreoacanthocytosis. Neurology. 41:1000-1006.

38. Redman, C.M., Huima, T., Robbins, E., Lee, S., and Marsh, W.L. 1989. Effect of phosphatidylserine on the shape of McLeod red cell acanthocytes. Blood. 74:1826-1835.

39. Brecher, G., and Bessis, M. 1972. Present status of spiculed red cells and their relationship to the discocyte-echinocyte transformation: a critical review. Blood. 40:333-344.

40. Barry, P.H., and Lynch, J.W. 1991. Liquid junction potentials and small cell effects in patch-clamp analysis (review). J. Membr. Biol. 121:101-117. 